\title{
Public Health Infos
}

https://doi.org/10.1515/pubhef-2019-0130

\section{Tagungen}

\section{Kokreative Werkstatt zur Neuorientierung des Dachverbandes Salutogenese e.V.}

\author{
„Wege zu einer neuen Gesundheitskultur"
}

27.-29.03.2020

Göttingen

Veranstalter: Dachverband Salutogenese e.V. (DachS e.V.)

Das ursprünglich für das erste Maiwochenende geplante DachS-Symposium des Dachverbands Salutogenese wird voraussichtlich im Herbst 2020 stattfinden. Nach Veränderungen im Vorstand des Dachverbandes findet stattdessen im ersten Halbjahr 2020 eine kokreative Werkstatt statt. Entsprechend der Gründungsintention des Dachverbandes - „Wir verstehen die salutogenetischen Orientierung als grundlegende handlungs- und erkenntnisleitende Perspektive“ (Präambel der Satzung) - soll dort die Ausgestaltung dieses Konsenses gemeinsam neu konzipiert werden. Es sollen Visionen, Erfahrung und organisatorische Umsetzung neu entwickelt werden. Die Veranstaltung wird voraussichtlich in der Kreisvolkshochschule Mariaspring in Göttingen stattfinden. Nähere Einzelheiten werden ab Dezember auf der Seite www.dachverband-salutogenese.de mitgeteilt.

\section{International Conference on Salutogensis}

\author{
„Advancing Salutogenesis towards thriving societies“ \\ 14.-15.04.2020 \\ Zürich, Schweiz
}

Veranstalter: International Union for Health Promotion and Education (IUHPE), Society for Theory and Research on Salutogenesis (STARS)

Ziel der 6. Internationalen Konferenz zur Salutogenese ist es, Theorie und Forschung zur Salutogenese weiterzuentwickeln sowie neue Ansätze zu teilen und zu diskutieren. Im Mittelpunkt stehen folgende vier Bereiche: die Weiterentwicklung des salutogenetischen Models, die Weiterentwicklung des Konzeptes des Kohärenzgefühls (Sense of Coherence, SoC), die Gestaltung salutogenetischer Intervention und die Frage, wie Salutogenese auch über den Gesundheitssektor hinaus angewandt werden kann. Informationen zur Konferenz sind auf der Seite www. stars-society.org zusammengestellt.

\section{Wissenschaftlicher Kongress des BVÖGD und des BZÖG}

„Der Öffentliche Gesundheitsdienst - Große
Gesundheitsziele fördern“

23.-25.04.2020

Saarbrücken

Veranstalter: Landesverband Saarland der Ärztinnen und Ärzte des ÖGD e.V., Bundesverband der Ärztinnen und Ärzte des Öffentlichen Gesundheitsdienstes e.V. (BVÖGD), Bundesverband der Zahnärzte des Öffentlichen Gesundheitsdienstes e.V. (BZÖG), in Zusammenarbeit mit der Gesellschaft für Hygiene, Umweltmedizin und Präventivmedizin (GHUP)

Der 70. Wissenschaftliche Kongress des BVÖGD und BZÖG findet vom 23.04. bis zum 25.04.2020 in Saarbrücken statt. Unter dem Motto „Große Gesundheitsziele fördern wir hier von klein auf“" wird die Umsetzung und Stimmigkeit von Gesundheitszielen auf Bund-, Länderund kommunaler Ebene diskutiert. Der jährliche Kongress dient der Stärkung der Rolle des ÖGD in diesem Prozess sowie der Förderung des Austauschs zwischen den Akteuren. Außerdem soll die Kooperation mit den Sozialmedizinischen Assistentinnen und Assistenten und den Hygieneinspektorinnen und -inspektoren verstetigt sowie die gute Zusammenarbeit mit der Gesellschaft für Hygiene, Umweltmedizin und Präventivmedizin (GHUP) fortgesetzt werden. Informationen zum Kongress sind auf der Seite www.bvoegd-kongress.de bereitgestellt.

\section{Hauptstadtkongress Medizin und Gesundheit 2020}

"Next Generation Healthcare: Steigerung von Qualität
und Effizienz"

17.-19.06.2020

Berlin

Veranstalter: WISO S.E. Consulting GmbH 
Unter dem Motto „Next Generation Healthcare: Steigerung von Qualität und Effizienz“ blickt der Hauptstadtkongress 2020 in die Zukunft des Gesundheitswesens. Es geht dabei nicht nur um medizinischen Fortschritt und Innovationen, denn mit verbesserter Medizin und neuen Technologien müssen sich auch Versorgungsstrukturen, Selbstverwaltungen, Zulassungsverfahren, Qualitätsmanagement und Nutzenbewertung verändern. Informationen zum Kongress, Programm und Anmeldung sind zu finden unter www.hauptstadtkongress.de.

\section{Related Links}

Das Potsdam-Institut für Klimaforschung (PIK) untersucht wissenschaftlich und gesellschaftlich relevante Fragestellungen in den Bereichen Globaler Wandel, Klimawirkung und Nachhaltige Entwicklung. Naturund Sozialwissenschaftler erarbeiten interdisziplinäre Einsichten, welche wiederum eine Grundlage für Entscheidungen in Politik, Wirtschaft und Zivilgesellschaft darstellen. Um die Zusammenhänge zwischen Klimaveränderungen und der Bevölkerungsgesundheit $\mathrm{zu}$ erforschen, hat die Charité - Universitätsmedizin Berlin gemeinsam mit dem Potsdam-Institut für Klimaforschung die bundesweit erste Professur für Klimawandel und Gesundheit an einer medizinischen Fakultät eingerichtet. Nicht nur die Folgen von Hitzewellen und die Ausbreitung tropischer Infektionskrankheiten sollen im Fokus stehen, sondern auch die enge Zusammenarbeit mit Agrarökonomen, um so die Wechselwirkung zwischen Landwirtschaft und Klimawandel zu untersuchen. Beispielweise soll erforscht werden, welchen Einfluss die Veränderung der Landwirtschaft auf die Ernährungsgewohnheiten und die Gesundheit verschiedener Bevölkerungsgruppen in Entwicklungs- und Schwellenländern hat. Nähere Informationen zum PIK selbst und zur Forschung des PIK sind auf der Seite www.pik-potsdam.de zu finden.

Die Deutsche Allianz Klimawandel und Gesundheit e.V. (KLUG) ist ein Netzwerk von Personen, Organisationen und Verbänden aus dem Gesundheitsbereich. Ziel ist es, die Folgen des Klimawandels auf die Gesundheit zu verdeutlichen. KLUG möchte erreichen, dass der Klimawandel von einer kritischen Menge von Akteurinnen und Akteuren als wichtige Herausforderung für den Gesundheitssektor anerkannt wird, dass Klimaschutz aktiv im Gesundheitswesen umgesetzt wird, damit gleichzeitig auch der damit verbundene Gesundheitsnutzen priorisiert wird, und dass der Gesundheitssektor Führung und Verantwortung übernimmt in der gesamtgesellschaftlichen Transformation $\mathrm{zu}$ einer klimaneutralen Gesellschaft. Die Internetseite www.klimawandel-gesundheit.de informiert über KLUG, über ihre Aktivgruppen, die regional bzw. themenspezifisch aktiv sind und über die Aktivitäten des Vereins. Die Webseite stellt Meldungen, Materialien und Argumentationshilfen zur Verfügung und stellt Linklisten zusammen zu Organisationen aus dem Gesundheitsbereich, die zum Thema Klimawandel und Gesundheit arbeiten, zu Bildungsangeboten, Forschungsprojekten und einführender bzw. zentraler Literatur sowie informativer Webseiten zum Thema Klima und Gesundheit.

Das Umweltbundesamt (UBA) ist Deutschlands zentrale Umweltbehörde. Hauptaufgabe ist die Schaffung oder Bewahrung einer gesunden Umwelt, in der Menschen so weit wie möglich vor schädlichen Umwelteinwirkungen geschützt leben können. Die Themenpalette reicht von der Abfallvermeidung über den Klimaschutz bis zur Zulassung von Pflanzenschutzmitteln. Auftrag des UBA ist es, Daten über den Zustand der Umwelt zu erheben, Zusammenhänge zu erforschen, Prognosen für die Zukunft zu erstellen und mit diesem Wissen die Bundesregierung $\mathrm{zu}$ beraten. Eine weitere Aufgabe ist es, die Öffentlichkeit in Umweltfragen zu informieren und Umweltgesetze umzusetzen. Das UBA versteht sich als Frühwarnsystem, das mögliche zukünftige Beeinträchtigungen des Menschen und der Umwelt rechtzeitig erkennt, bewertet und praktikable Lösungen vorschlägt. In Forschungsprojekten werden Erkenntnisse gewonnen über Herkunft und Ausmaß von gesundheitlich relevanten Umweltbelastungen. Im fachübergreifenden Ansatz arbeiten hier verschiedene Bereiche der Hygiene, der Medizin, der Naturwissenschaften und der Epidemiologie zusammen. Sie analysieren, wie stark die Schadstoffbelastungen des Körpers sind und kombinieren dieses Wissen mit Daten der Verunreinigung von Wasser, Boden und Luft. Das Risiko von umweltbedingten Gesundheitsbelastungen wird bewertet, indem Informationen aus Human Biomonitoring, Umweltmedizin, Toxikologie und Mikrobiologie gemeinsam ausgewertet werden. Das UBA stellt auf www.umweltbundesamt.de/themen sowohl im Bereich Klima also auch im Bereich Gesundheit und Informationen, Publikationen und weiterführende Links zur Verfügung.

Ziel der Weltgesundheitsorganisation (WHO) ist es, für alle Völker das höchstmögliche Gesundheitsniveau zu erreichen. Mit ihren 194 Mitgliedstaaten ist sie federführend in globalen Gesundheitsfragen und in der Gestaltung der Forschungsagenda für Gesundheit, im Aufstellen von Normen und Standards und in der Formulierung 
evidenzbasierter Grundsatzoptionen. Die WHO bietet ihren Mitgliedstaaten fachliche Unterstützung, sie überwacht und bewertet gesundheitliche Entwicklungen, sie unterstützt medizinische Forschung und leistet Soforthilfe bei Katastrophen. Sie setzt sich weltweit für bessere Ernährung und für eine Verbesserung der Wohn- und Arbeitsbedingungen sowie der sanitären Verhältnisse ein. Sowohl die Seite der WHO international also auch die Seite der europäischen Abteilung der Organisation unterhalten Unterseiten zum Klimawandel: www.euro.who. int/en/health-topics/environment-and-health/Climatechange und www.who.int/health-topics/climate-change. Beide Seiten informieren über diesbezügliche Neuigkeiten, Daten und Statistiken, Publikation, Projekte und politische Aktivitäten.

\section{Weiterführende Literatur}

WHO, Herausgeber. Gesündere und glücklichere Städte für alle. Ein transformativer Ansatz für sichere, inklusive, nachhaltige und widerstandsfähige Gesellschaften. Kopenhagen: WHO, 2018.

Krämer A, Wörmann T, Jahn HJ. Klimawandel und Gesundheit: Grundlagen und Herausforderungen für den Public Health-Sektor. In: Jahn HJ, Krämer A, Wörmann T, Hrsg. Klimawandel und Gesundheit. Internationale, nationale und regionale Herausforderungen und Antworten. Berlin: Springer Verlag, 2013:1-21.

United Nations Development Programme (UNDP). Human Development Report 2011. Sustainability and Equity: a better future for all. New York, USA: Palgrave, 2011.

World Health Organization (WHO), Herausgeber. World Health Statistics 2014. Genf, Schweiz: WHO, 2014.

Umweltbundesamt, Hrsg. Vulnerabilität Deutschlands gegenüber dem Klimawandel. Dessau-Roßlau: Umweltbundesamt, 2015.

Agustin J, Sauerborn R, Burkart K, Endlicher W, Jochner S, Koppe C, et al. Gesundheit. In: Brasseur GP, Jacob D, Schuck-Zöller S, Hrsg. Klimawandel in Deutschland. Entwicklung, Folgen und Perspektiven. Heidelberg, Berlin: Springer, 2017:137-49.

Austrian Panel on Climate Change (APCC). Health, Demography and Climate Change. Austrian Special Report (ASR) 18. Vienna: Austrian Academy of Sciences Press, 2018.

Bundesministerium für Nachhaltigkeit und Tourismus. Die österreichische Strategie zur Anpassung an den Klimawandel. Teil 2 - Aktionsplan. Aktualisierte Fassung. Wien, 2017.
Brasseur GP, Jacob D, Schuck-Zöller S, Hrsg. Klimawandel in Deutschland. Entwicklung, Folgen und Perspektiven. Heidelberg, Berlin: Springer, 2017:225-34.

Austrian Panel on Climate Change (APCC). Health, Demography and Climate Change. Austrian Special Report (ASR) 18. Vienna: Austrian Academy of Sciences Press, 2018.

Knutson, T. Appendix C: Detection and Attribution Methodologies Overview. Climate Science Special Report: Fourth National Climate Assessment, Volume I. U.S. Global Change Research Program. Washington, DC, 2017.

World Health Organisation (WHO). Climate change and health. 2018.

\section{Forschung}

\section{Forschungsprojekt HOPE - Die Rolle der Gesundheit bei klimafreundlichem Verhalten}

In dem interdisziplinären Projekt HOPE (Household Preference for reducing greenhouse gas Emissions) wurde in vier europäischen Städten in Deutschland (Mannheim), Frankreich (Aix-en-Provence), Norwegen (Bergen) und Schweden (Umeå) untersucht, welche Präferenzen Haushalte bei der Umsetzung von Klimaschutzmaßnahmen haben.

Hintergrund ist, dass viele Lebensstil-assoziierte Maßnahmen zum Klimaschutz auch direkt zum Gesundheitsschutz beitragen. Dies trifft insbesondere auf die Bereiche Wohnen, Mobilität und Ernährung zu. So zeigen wissenschaftliche Studien, dass nicht nur Fahrrad fahren und laufen, sondern auch die Nutzung öffentlicher Verkehrsmittel die Gesundheit im Vergleich zur PKW-Nutzung fördern. Auch eine Reduktion des Fleischkonsums bedeutet bei adäquatem Ersatz durch pflanzliche Eiweiße eine klimafreundlichere und gesündere Lebensweise. Gute Isolierung von Gebäuden kann insbesondere bei älteren Menschen die Hospitalisierungsraten verringern. Man bezeichnet solche Effekte als gesundheitliche Co-Benefits von Klimaschutzmaßnahmen.

Im HOPE Projekt wurde quantitativ und qualitativ untersucht, inwiefern Informationen $\mathrm{zu}$ gesundheitlichen Co-Benefits die Bereitschaft von Haushalten beeinflussen, Klimaschutzmaßnahmen umzusetzen. Quantitativ konnte im Rahmen eines experimentellen Ansatzes gezeigt werden, dass Teilnehmende in den Bereichen Wohnen und Ernährung, nicht jedoch im Bereich Mobilität, eine größere Bereitschaft zeigten, ihren $\mathrm{CO}_{2}$-Fußabdruck $\mathrm{zu}$ reduzieren, wenn sie 
Informationen $\mathrm{zu}$ gesundheitlichen Co-Benefits erhielten. Unter zu Hilfenahme der qualitativen Ergebnisse zeigte sich, dass Haushalte im Bereich der Mobilität zwar die gesundheitlichen Vorteile erkennen, dass hier aber die Hindernisse zur Umsetzung besonders groß sind. Dies bedeutet, dass einerseits Informationen $\mathrm{zu}$ gesundheitlichen Co-Benefits von Klimaschutzmaßnahmen kommuniziert, aber auch strukturelle Hürden für deren Umsetzung abgebaut werden müssen. Es braucht zur Förderung gesünderer und klimafreundlicherer Lebensstile also eine Kombination aus Verhaltens- und Verhältnisprävention.

Zuletzt wurden die Ergebnisse zur Rolle der Gesundheit hierbei veröffentlicht:

- Übersicht: https://link.springer.com/article/10.1007\%2 Fs00103-019-02929-7

- Quantitative Ergebnisse: https://www.sciencedirect. com/science/article/pii/S095937801830551X

Obwohl die gesundheitlichen Co-Benefits von Klimaschutzmaßnahmen große Synergien zwischen Klimaund Gesundheitsschutz versprechen und in der globalen Public Health-Forschung wachsende Aufmerksamkeit erhalten, werden sie in der deutschen Forschungs- und Versorgungslandschaft noch zu wenig berücksichtigt.

Kontakt: Dr. med. Alina Herrmann, Heidelberger Institut für Global Health, Universitätsklinikum Heidelberg, alina.herrmann@uni-heidelberg.de

\section{Zentrum für öffentliches Gesundheitswesen und Versorgungsforschung Tübingen gegründet}

Am 2. Oktober 2019 wurde am Universitätsklinikum Tübingen (UKT) das Zentrum für öffentliches Gesundheitswesen und Versorgungsforschung Tübingen (ZÖGV) gegründet. Ziel des Zentrums ist es, die Universitätsmedizin stärker mit der Versorgungsforschung und dem öffentlichen Gesundheitswesen (ÖGW) zu verzahnen. Es sollen Versorgungsforschungsprojekte im ÖGW initiiert und bearbeitet, Methoden der Versorgungsforschung vertieft und so beide Bereiche gestärkt werden.

Den Wunsch ein solches Zentrum am UKT zu gründen, gab es bereits seit 2015, wie im Rahmen der Eröffnung des Zentrums dargestellt wurde. Im Mittelpunkt steht die Bevölkerungsperspektive. Dabei spielen auch die Gesundheitsförderung und Prävention eine wesentliche Rolle. Der Fokus medizinischer Fakultäten richtete sich bisher überwiegend auf die Grundlagen- und klinische
Forschung. Anspruch an die zukünftige Universitätsmedizin ist es, den Bogen weiter zu spannen und die gesamte Gesundheitsversorgung zu betrachten, so dass neben der biomedizinischen Forschung die Versorgungsforschung immer wichtiger wird. Bei dieser Translation setzt das Zentrum mit seinen Zielen an.

Die $\mathrm{zu}$ bearbeitenden Themen sind mannigfaltig und reichen von Infektions- und Impfschutz, Prävention, Herz-Kreislauf- und Tumorerkrankungen, Klimawandel, regionale Versorgungsplanung bis zur Notfallversorgung der Bevölkerung und Einbindung in Gesundheitssysteme sowie Berücksichtigung technischer Entwicklungen, wie zum Beispiel Telemedizin. In den zahlreichen Redebeiträgen von Vertretern der Universität, des Universitätsklinikums, der kommunalen Ebene und des öffentlichen Gesundheitswesens sowie der begleitenden umfangreichen Posterpräsentation wurde deutlich, wie vielfältig die $\mathrm{zu}$ bearbeiteten wissenschaftlichen Projekte im Bereich der Versorgungsforschung sind und wie fruchtbar die ersten Kooperationsprojekte zwischen ÖGD und Wissenschaft bereits verlaufen.

Wünsche, die im Rahmen der Veranstaltung aus dem Auditorium geäußert wurden, betrafen die niederschwellige Nutzung der wissenschaftlichen Expertise der Universitäten und eine partnerschaftliche Zusammenarbeit der Wissenschaft mit den Gesundheitsämtern und allen Akteuren des Öffentlichen Gesundheitswesens. Als weiterer Wunsch an das neue Zentrum wurde geäußert, als Moderator und Impulsgeber zu fungieren und eine akademische Anbindung des Öffentlichen Gesundheitsdienstes in Forschung und Lehre zu schaffen. Zentrale Anliegen seien, die Attraktivität des ÖGD für junge Mediziner zu steigern, den evidenz- und wertebasierten Austausch sowie eine interprofessionelle Forschung und Lehre, die alle Heilberufe anspricht, zu fördern.

Weitere Anregungen und Wünsche der einzelnen Vertreter und Repräsentanten an das ZÖGV sollen in den kommenden Monaten systematisch erfasst, diskutiert und priorisiert werden, um dann in konkrete gemeinsame Forschungsprojekte zu münden.

Weitere Informationen: www.medizin.uni-tuebingen. de/versorgungsforschung/

\section{Kontakt:}

Dr. David Häske, MSc MBA

Zentrum für Öffentliches Gesundheitswesen und Versorgungsforschung Tübingen

c/o Institut für Arbeitsmedizin, Sozialmedizin und Versorgungsforschung

Wilhelmstr. 27, 72074 Tübingen

david.haeske@med.uni-tuebingen.de 